

\title{
Feasibility Study of Mobius3D for Patient-Specific Quality Assurance in the Volumetric Modulated Arc Therapy
}

\author{
Chang Yeol Lee ${ }^{\mathbb{D}}$, Woo Chul Kim ${ }^{\mathbb{1}}$, Hun Jeong Kim ${ }^{\mathbb{1}}$, Jeongshim Lee ${ }^{\mathbb{1}}$, Hyun Do Huh ${ }^{\mathbb{1}}$ \\ Department of Radiation Oncology, Inha University College of Medicine, Incheon, Korea
}

Received 15 November 2019 Revised 26 November 2019

Accepted 27 November 2019

\section{Corresponding author}

Hyun Do Huh

(hyundohuh@gmail.com)

Tel: 82-32-890-3073

Fax: 82-32-890-3082
Purpose: This study was designed to evaluate the dosimetric performance of Mobius3D by comparison with an aSi-based electronic portal imaging device (EPID) and Octavius 4D, which are conventionally used for patient-specific prescription dose verification.

Methods: The study was conducted using nine patients who were treated by volumetric modulated arc therapy. To evaluate the feasibility of Mobius3D for prescription dose verification, we compared the QA results of Mobius3D to an aSi-based EPID and the Octavius 4D dose verification methods. The first was the comparison of the Mobius3D verification phantom dose, and the second was to gamma index analysis.

Results: The percentage differences between the calculated point dose and measurements from a PTW31010 ion chamber were $1.6 \% \pm 1.3 \%, 2.0 \% \pm 0.8 \%$, and $1.2 \% \pm 1.2 \%$, using collapsed cone convolution, an analytical anisotropic algorithm, and the AcurosXB algorithm respectively. The average difference was found to be $1.6 \% \pm 0.3 \%$. Additionally, in the case of using the PTW31014 ion chamber, the corresponding results were $2.0 \% \pm 1.4 \%, 2.4 \% \pm 2.1 \%$, and $1.6 \% \pm 2.5 \%$, showing an average agreement within $2.0 \% \pm 0.3 \%$. Considering all the criteria, the Mobius $3 \mathrm{D}$ result showed that the percentage dose difference from the EPID was within $0.46 \% \pm 0.34 \%$ on average, and the percentage dose difference from Octavius $4 \mathrm{D}$ was within $3.14 \% \pm 2.85 \%$ on average.

Conclusions: We conclude that Mobius3D can be used interchangeably with phantom-based dosimetry systems, which are commonly used as patient-specific prescription dose verification tools, especially under the conditions of $3 \% / 3 \mathrm{~mm}$ and $95 \%$ pass rate.

Keywords: Mobius3D, Patient-specific quality assurance, Volumetric modulated arc therapy, Gamma index analysis

\section{Introduction}

In the 1990s, the development of linear accelerators and treatment planning, which makes possible three dimentional (3D) dose calculation using computed tomography (CT) images, led to the new radiotherapy method of threedimensional conformal radiotherapy (3DCRT). Since then, various radiotherapy techniques such as intensity modu- lated radiation therapy (IMRT), volumetric modulated arc therapy (VMAT), and stereotactic radiosurgery have been developed and have become available for treatment. ${ }^{1-3)}$ In particular, VMAT is a radiotherapy in which the treatment device rotates $360^{\circ}$ around the patient at high speed while adjusting the radiation rate, gantry speed, and irradiation shape in real time. The method minimizes the amount of radiation distributed around the surrounding normal tis- 
sues whilst delivering sufficient irradiation to kill cancer cells. $^{4)}$

Complex and sophisticated treatment techniques also increase the possibility of errors in patient treatment and treatment planning compared with the conventional 3DCRT, thus requiring a precise and strict quality assurance program. ${ }^{5)}$ The quality assurance program should be implemented efficiently and effectively. In addition, patient-specific prescription dose verification should be performed to verify that the radiation is delivered according to the treatment plan. The TASK GROUP 120 of the American Association of Physicists in Medicine sets out the measurement procedures and dose criteria for various tools used for the verification of patient-specific prescribed doses from IMRT. The report addresses the need for relative dose measurements in a given area or volume, as well as point dose measurements, for patient-specific prescription dose verification. ${ }^{6}$ For example, many researchers have investigated and reported on two dimentional (2D) and 3D dose analysis using 2D array detectors, electronic portal imaging devices (EPIDs), gels, and films. ${ }^{7-10)}$

Recently, Mobius3D (developed by Mobius Medical Systems, Houston, TX, USA), which can verify a patientspecific prescribed dose by a treatment log-file-based method rather than a phantom-based method, has been used commercially. This dosimetry system can verify treatment plans as well as the prescribed dose using a 3D dose calculation algorithm independent of the treatment logfile. ${ }^{11)}$ The main advantage of this dosimetry system is that a user does not have to consider the setup errors involved in the installation of a dosimetry device and is not subject to the temporal and spatial constraints of a physical dosimetry device. Therefore, this study was designed to evaluate the dosimetric performance of Mobius3D by comparison with the QA results of an aSi-based EPID (aS1200; Varian Medical Systems, Palo Alto, CA, USA) and Octavius 4D (PTW Freiburg GmbH, Freiburg, Germany), which are conventionally used for patient-specific prescription dose verification. The study was conducted using nine patients, who were treated by volumetric modulated arc therapy.

\section{Materials and Methods}

\section{Patient-specific quality assurance system}

The patient-specific prescription dose verification systems used in this study to verify the treatment plan of VMAT therapy were Mobius3D, EPID, and Octavius 4D. Mobius3D largely consists of two parts. First, MobiusCalc performs secondary confirmation of the treatment plan, in which the recalculation of doses is based on the collapsed cone convolution algorithm using the CT data of the patients and the treatment plan parameters. The second part is MobiusFx, which uses the Mobius3D beam model and treatment log-files from the treatment device to verify the dose delivered to the patient from each treatment. A treatment log-file provides beam delivery parameters such as the location of the multi-leaf collimator, gantry rotation speed, and dose rate with a temporal resolution of $50 \mathrm{~ms}^{12)}$

The Mobius3D beam model is created primarily using accelerator-specific universal beam data, provided by Mobius Medical Systems (the manufacturer of Mobius3D). While this model can be customized to better fit the user's individual treatment machine, Mobius Medical Systems recommends minimizing the application of beam data for the user's equipment to the Mobius3D system, to maintain Mobius3D's independence as a secondary system to confirm treatment plans. Therefore, in this study, the application of user beam data to the Mobius3D system has been minimized, following the recommendations of the manufacturer. The aSi-based EPID used in this study has an ar-

Table 1. Characteristics for plans used in this study

\begin{tabular}{lccccc}
\hline $\begin{array}{c}\text { Treatment } \\
\text { site }\end{array}$ & $\begin{array}{c}\text { Plan } \\
\text { number }\end{array}$ & $\begin{array}{c}\text { Average field } \\
\text { size }\left(\mathrm{cm}^{2}\right)\end{array}$ & $\begin{array}{c}\text { No. of } \\
\text { segments }\end{array}$ & $\begin{array}{c}\text { Total } \\
\text { MU }\end{array}$ & $\begin{array}{c}\text { PTV doses } \\
\text { (cGy) }\end{array}$ \\
\hline Head and & 1 & $16.5 \times 17.8$ & 228 & 691 & 200 \\
neck & 2 & $18.4 \times 18.3$ & 356 & 633 & 212 \\
& 3 & $19.2 \times 19.9$ & 356 & 606 & 220 \\
Lung & 4 & $11.9 \times 11.8$ & 228 & 436 & 200 \\
& 5 & $19.0 \times 10.0$ & 356 & 449 & 200 \\
Prostate & 7 & $9.8 \times 10.0$ & 196 & 719 & 300 \\
& 8 & $15.8 \times 15.9$ & 356 & 506 & 180 \\
& 9 & $14.0 \times 14.9$ & 356 & 631 & 180 \\
\hline
\end{tabular}

MU, monitor unit; PTV, planning target volume. 
ray of $1,280 \times 1,280$ pixels and an active image area of $43 \times 43$ $\mathrm{cm}$. Measured doses were analyzed using EPID-dedicated software. The Octavius $4 \mathrm{D}^{\circledR} 1500$ system consists of an Octavius 4D modular phantom (32 $\mathrm{cm}$ in diameter and 34.3 $\mathrm{cm}$ in length) and a 2D array of 1405 vented ion chambers (active area of $27 \times 27 \mathrm{~cm}^{2}$ ). Verisoft (v.7.1) software (PTW Freiburg $\mathrm{GmbH}$ ) was used for measurement with perpendicular for every gantry angle, and 3D dose matrices of voxel size $2.5 \times 2.5 \times 2.5 \mathrm{~mm}$ were reconstructed.

\section{Treatment plan selection and delivery}

Nine patients with head and neck, lung, and prostate cancer (3 for each treatment site), who underwent VMAT treatment with a VitalBeam linear accelerator (Varian Medical Systems), were selected (Table 1). The dose calculation for all treatment plans was performed using Eclipse v.15.5. To verify the prescribed dose of the calculated treatment

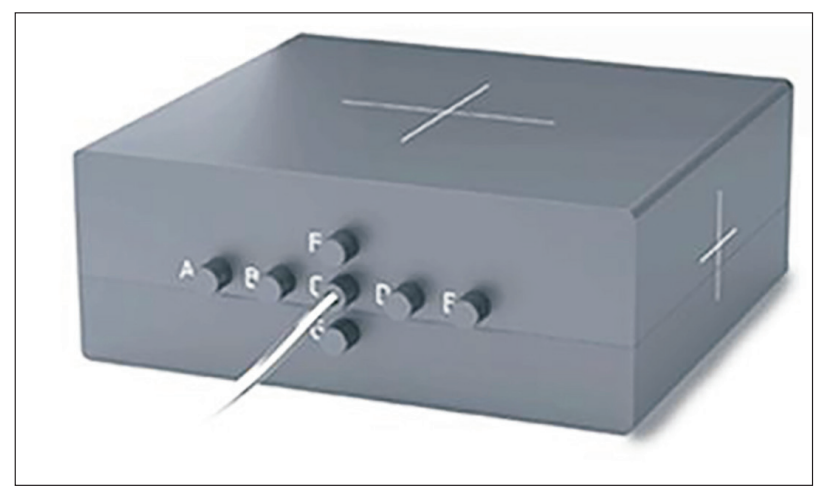

Fig. 1. Image of the mobius verification phantom with seven available ionization chamber positions. plan, a dose verification method for each patient-specific prescription dose verification system was implemented. To examine inter-fraction variation, five fractions were randomly selected during the entire treatment period to verify the prescription dose.

\section{MVP dose comparison and gamma evaluation}

To evaluate the feasibility of Mobius3D in the prescription dose verification, we compared the QA results of the aSi-based EPID and Octavius 4D dose verification methods. The first comparison was to the Mobius3D Verification Phantom (MVP) dose, and the second comparison was to gamma index analysis. MVP dose comparison can measure point doses at seven measurement points using several ion chambers (Fig. 1). The measurement point selected in this study was the $C$ point, located in the center of the phantom, and the PTW31010 and PTW31014 models were selected as the ion chambers. The PTW31010 ion chamber has a nominal sensitive volume of $0.125 \mathrm{~cm}^{3}$, while the PTW31014 has $0.015 \mathrm{~cm}^{3}$. Mobius3D provides a collapsed cone convolution (CC) algorithm, and Eclipse provides two algorithms, which are the analytical anisotropic algorithm (AAA) and Acuros XB (Acuros XB advanced dose calculation algorithm). For each algorithm, the differences in dose distribution were compared. The criteria applied to the gamma index analysis were $2 \% / 2 \mathrm{~mm}, 2 \% / 3 \mathrm{~mm}, 3 \% / 2$ $\mathrm{mm}$, and $3 \% / 3 \mathrm{~mm}$; the criteria were applied to 45 fractions in total.

Table 2. Comparison of MVP dose calculated by three dose calculation algorithms at point $\mathrm{C}$ and measured by two ionization chambers

\begin{tabular}{|c|c|c|c|c|c|c|}
\hline \multirow{2}{*}{$\begin{array}{l}\text { Treatment } \\
\text { site }\end{array}$} & \multirow{2}{*}{$\begin{array}{c}\text { Plan } \\
\text { number }\end{array}$} & \multicolumn{3}{|c|}{ Dose calculation algorithm } & \multicolumn{2}{|c|}{ Ionization chambers for measurement } \\
\hline & & CC (cGy) & AAA (cGy) & AcurosXB (cGy) & PTW 31010 (cGy) & PTW 31014 (cGy) \\
\hline \multirow{3}{*}{$\begin{array}{l}\text { Head and } \\
\text { neck }\end{array}$} & 1 & 200.5 & 201.0 & 203.6 & 207.5 & 207.6 \\
\hline & 2 & 230.7 & 229.8 & 234.7 & 235.5 & 234.5 \\
\hline & 3 & 225.7 & 226.3 & 228.2 & 227.7 & 232.6 \\
\hline \multirow[t]{3}{*}{ Lung } & 4 & 256.6 & 251.7 & 254.2 & 255.9 & 255.6 \\
\hline & 5 & 279.4 & 282.4 & 287.5 & 288.0 & 284.5 \\
\hline & 6 & 374.1 & 368.9 & 372.8 & 373.7 & 373.7 \\
\hline \multirow[t]{3}{*}{ Prostate } & 7 & 351.2 & 354.3 & 358.1 & 361.2 & 360.2 \\
\hline & 8 & 108.6 & 105.1 & 104.5 & 108.1 & 113.6 \\
\hline & 9 & 124.7 & 124.2 & 123.5 & 126.9 & 123.3 \\
\hline
\end{tabular}

MVP, mobius verification phantom; CC, collapsed cone; AAA, analytical anisotrophic algorithm. 


\section{Results}

\section{MVP dose comparison}

Table 2 shows the results of three dose calculation algorithms computed at point $\mathrm{C}$ of the MVP and measurement results from the two PTW ion chambers. Fig. 2 shows the relative percentage dose difference between the results of the dose calculation algorithms and the measured values from each ion chamber. The average measured dose difference between the two chambers was $1.3 \% \pm 1.7 \%$. However, the results of the calculated value showed that the difference in the result calculated by the three algorithms was found to be consistent with the average within $1.1 \%$ with AAA and $1.7 \%$ with AcurosXB, having the CC algorithm as a reference.

The differences between the calculated values and the measured values from the PTW31010 ion chamber were $1.6 \% \pm 1.3 \%, 2.0 \% \pm 0.8 \%$, and $1.2 \% \pm 1.2 \%$, for the CC, AAA, and the AcurosXB algorithms respectively, showing an average agreement within $1.6 \% \pm 0.3 \%$. Using the PTW31014 ion chamber the corresponding differences were $2.0 \% \pm 1.4 \%, 2.4 \% \pm 2.1 \%$, and $1.6 \% \pm 2.5 \%$, showing an average agreement within $2.0 \% \pm 0.3 \%$.

Table 3 shows the differences between calculated values from Mobius3D and the measured values from different a

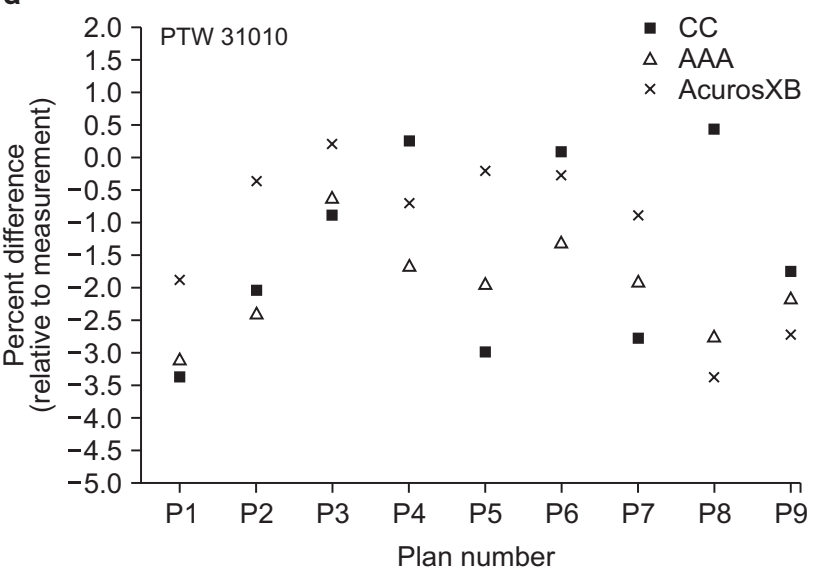

b

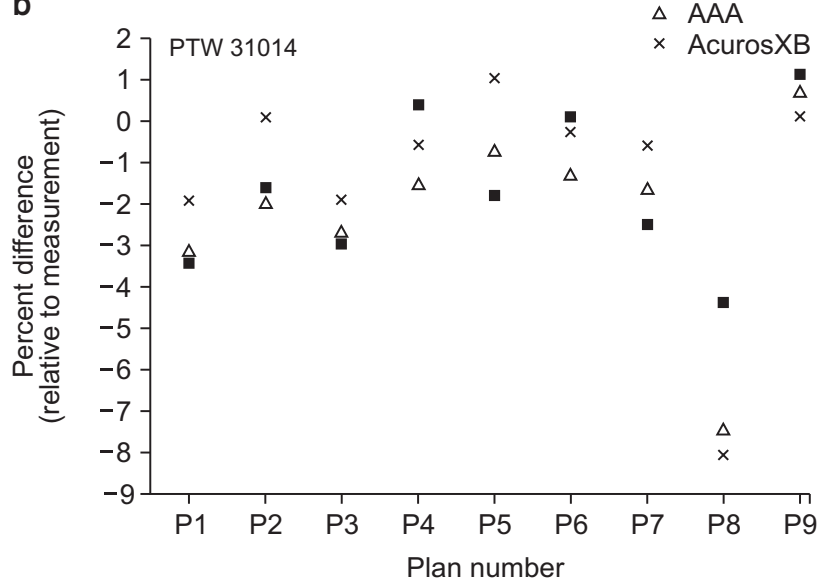

Fig. 2. Percentage differences between dose calculated with (a) Eclipse AAA and Mobius3D, and dose measured with PTW 31010 in the MVP (b) Eclipse AcurosXB and Mobius3D, and dose measured with PTW 31014 in the MVP. MVP, mobius verification phantom; CC, collapsed cone; AAA, analytical anisotrophic algorithm.

Table 3. Comparison of this study to other selected publications evaluating Mobius3D vs. ionization chamber measurements

\begin{tabular}{|c|c|c|c|c|c|c|}
\hline Reference & $\begin{array}{l}\text { Delivery } \\
\text { technique }\end{array}$ & $\begin{array}{l}\text { Number of } \\
\text { plans analyzed }\end{array}$ & $\begin{array}{l}\text { Points } \\
\text { analyzed } \\
\text { per plan }\end{array}$ & $\begin{array}{c}\text { Ionization } \\
\text { chamber }\end{array}$ & Phantom & $\begin{array}{c}\text { Reported } \\
\text { average percent } \\
\text { difference } \pm \text { standard } \\
\text { deviation }(\%)\end{array}$ \\
\hline \multirow[t]{2}{*}{ Nelson et al. ${ }^{13)}$} & VMAT & 12 & \multirow[t]{2}{*}{1} & \multirow[t]{2}{*}{ IBA CC04 } & \multirow{2}{*}{$\begin{array}{l}\text { Rectangular } \\
\text { solid water }\end{array}$} & $1.5 \pm 1.0$ \\
\hline & IMRT & 28 & & & & $-0.2 \pm 1.0$ \\
\hline \multirow[t]{2}{*}{ Fontenot $^{14)}$} & VMAT & 4 & \multirow[t]{2}{*}{$2-3$} & \multirow{2}{*}{$\begin{array}{l}\text { Standard } \\
\text { imaging A1SL }\end{array}$} & \multirow{2}{*}{$\begin{array}{l}\text { Cylindrical } \\
\text { solid water }\end{array}$} & $-1.6 \pm 2.3$ \\
\hline & IMRT & 4 & & & & $-0.6 \pm 2.8$ \\
\hline $\begin{array}{l}\text { Clemente-Gutiérrez } \\
\text { et al. }^{15)}\end{array}$ & VMAT & 4 & 1 & IBA CC04 & IBA easycube & $0.9 \pm 1.7$ \\
\hline \multirow[t]{2}{*}{ McDonald et al. ${ }^{16)}$} & VMAT & 9 & \multirow[t]{2}{*}{2} & \multirow{2}{*}{$\begin{array}{l}\text { Standard } \\
\text { imaging AlSL }\end{array}$} & \multirow[t]{2}{*}{ Mobius MVP } & $0.2 \pm 1.3$ \\
\hline & IMRT & 8 & & & & $-0.7 \pm 1.0$ \\
\hline \multirow[t]{2}{*}{ Present study } & VMAT & 9 & 1 & PTW 31010 & Mobius MVP & $1.6 \pm 1.3$ \\
\hline & & & & PTW 31014 & & $2.0 \pm 1.4$ \\
\hline
\end{tabular}

VMAT, volumetric modulated arc therapy; IMRT, intensity modulated radiation therapy; MVP, mobius verification phantom. 
researchers using various phantoms and ion chambers. ${ }^{13-16)}$ In a study by McDonald et al. ${ }^{16)}$, which used the same Mobius MVP, an ion chamber with a volume of $0.053 \mathrm{~cm}^{3}$ was used. This chamber represents an intermediate volume between the volumes of the ion chambers used in this study, and the reported results are better than those of this study. The volume-averaging effect of the ion chamber was considered to be maximized in that case, and the results of this study agree with those reported by other researchers within $2 \%$, on average.

\section{Gamma evaluation}

Table 4 shows the results of gamma analysis using three prescription dose verification tools for five randomly selected VMAT courses on three treatment sites. The criteria applied to the gamma index analysis were $2 \% / 2 \mathrm{~mm}, 2 \% / 3$ $\mathrm{mm}, 3 \% / 2 \mathrm{~mm}$, and $3 \% / 3 \mathrm{~mm}$, and the analyzed threshold dose was $10 \%$. When the criteria was $2 \% / 2 \mathrm{~mm}$, the average pass rates were $97.2 \% \pm 1.0 \%, 97.9 \% \pm 1.6 \%$, and $91.1 \% \pm 2.2 \%$ for Mobius3D, EPID, and Octavius 4D, respectively. When the criteria was $2 \% / 3 \mathrm{~mm}$, the average pass rates were $99.3 \% \pm 0.4 \%, 98.7 \% \pm 1.0 \%$, and $95.4 \% \pm 1.6 \%$ for Mobius3D, EPID, and Octavius $4 \mathrm{D}$, respectively. When the criteria was $3 \% / 2 \mathrm{~mm}$, the average pass rates were $99.0 \% \pm 0.5 \%$, $99.0 \% \pm 0.9 \%$, and $97.1 \% \pm 1.1 \%$ for Mobius3D, EPID, and Octavius $4 \mathrm{D}$, respectively. Finally, when the criterion was $3 \% / 3$ $\mathrm{mm}$, the average pass rates were $99.8 \% \pm 0.2 \%, 99.47 \% \pm 0.6 \%$, and $99.1 \% \pm 0.4 \%$ for Mobius3D, EPID, and Octavius $4 \mathrm{D}$, respectively.

We applied the $3 \% / 3 \mathrm{~mm}$ and $95 \%$ pass rate condition, which are commonly applied criteria to the gamma index analysis. It was shown that the results from all three prescription dose verification tools satisfied the criteria. However, as the criteria were made stricter, the cases satisfying the $95 \%$ pass rate criteria were considerably reduced, particularly in the case of Octavius $4 \mathrm{D}$ where the mean pass rates decreased rapidly (Fig. 3). In addition, the mean standard deviations of the three patient-specific prescription dose verification tools tended to decrease gradually in the order of Octavius 4D, EPID, and Mobius3D.

When the gamma criteria were $2 \% / 2 \mathrm{~mm}, 2 \% / 3 \mathrm{~mm}$, $3 \% / 2 \mathrm{~mm}$, and $3 \% / 3 \mathrm{~mm}$, the relative percentage dose dif-

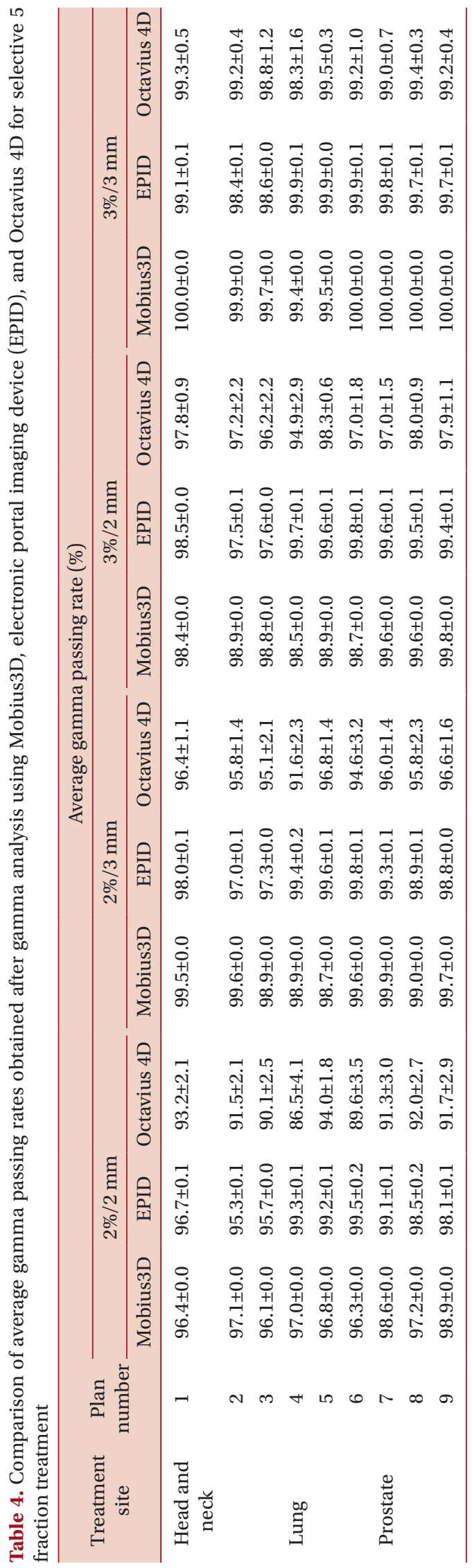



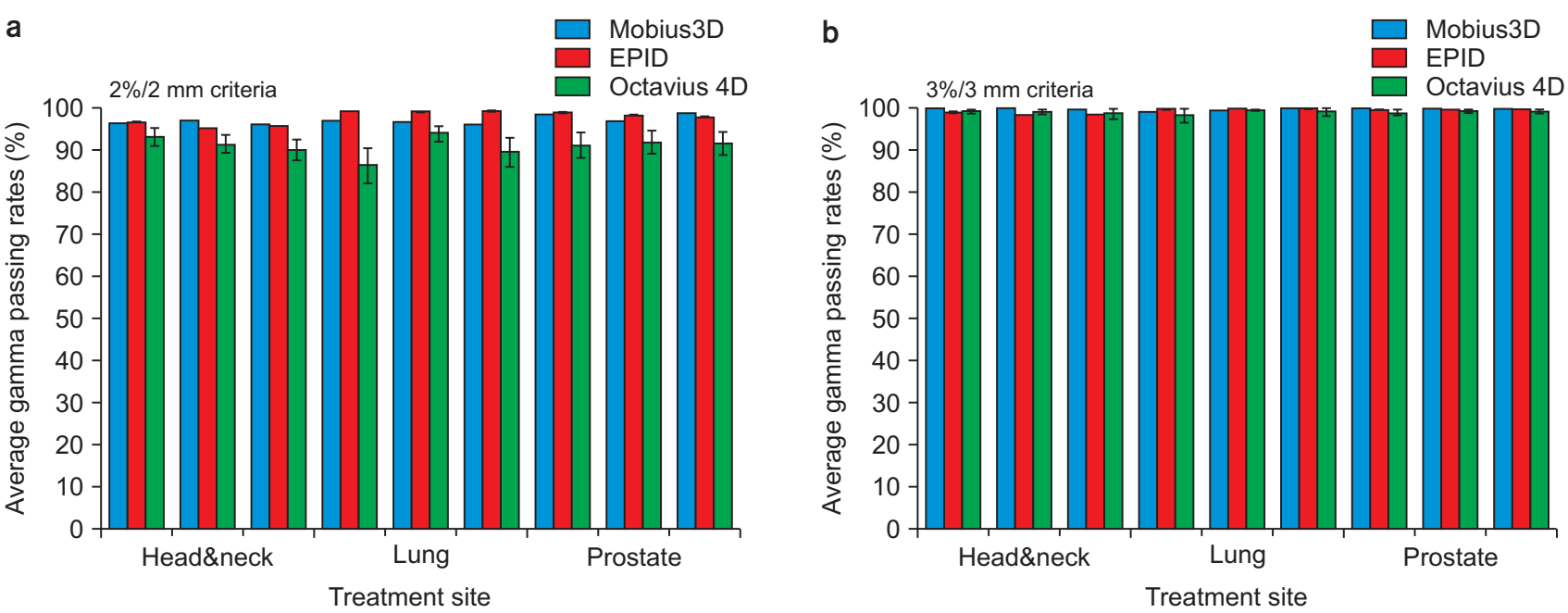

Fig. 3. Comparison histogram for average gamma passing rates with (a) $2 \% / 2 \mathrm{~mm}$, and (b) $3 \% / 3 \mathrm{~mm}$ criteria using Mobius3D, electronic portal imaging device (EPID), and Octavius 4D for head and neck, lung and prostate.

ferences between EPID and Mobius3D were $0.80 \%,-0.62 \%$, $0.00 \%$, and $-0.42 \%$, respectively. When the gamma criteria were $2 \% / 2 \mathrm{~mm}, 2 \% / 3 \mathrm{~mm}, 3 \% / 2 \mathrm{~mm}$, and $3 \% / 3 \mathrm{~mm}$, the relative percentage dose differences between Octavius $4 \mathrm{D}$ and Mobius3D were $-6.98 \%,-3.34 \%,-1.91 \%$, and $-0.31 \%$, respectively. Considering all the criteria, the Mobius3D results showed that the average percentage dose difference from the EPID was $0.46 \% \pm 0.34 \%$ and the average percentage dose difference from Octavius $4 \mathrm{D}$ was $3.14 \% \pm 2.85 \%$.

\section{Discussion}

In this study, the feasibility of Mobius3D as a patientspecific prescription dose verification tool was evaluated. Plan 8 showed a large difference of $4.8 \%$ between the two ion chambers' measurement values, and the difference between the calculated and the measured values was also large compared with other plans. However, the difference between the value measured by the PTW31010 ion chamber for Plan 8 and the value calculated by the CC dose calculation algorithm was within $0.5 \%$, indicating reasonable consistency. When measuring the dose using the ion chamber, it is important to properly select the location of the ion chamber. In most cases, when the ion chamber is located in an area with severe dose gradients, the dose error between the treatment plan results and the measurement results tends to be large. In the case of Plan 8 , the ion chamber was located in a region of severe dose gradient, which is thought to have caused the large difference between the calculated and measured values.

The volume-averaging effect corresponding to the size of the ion chamber should be considered for more accurate dose evaluation. An ion chamber with a small volume, such as the PTW31014, has a small output signal and a large signal-to-noise ratio, which may be a key factor affecting the dose error in the measurement of the absolute dose. However, the PTW31010 ion chamber, which has a larger volume than the PTW31014, had a large output signal, which can more accurately measure the dose.

The order of the dose calculation algorithms from smallest to largest difference between the measured and calculated values was AcurosXB, CC, and AAA. It is considered that, in the point dose measurement with the MVP, measurements using the PTW31010 ion chamber rather than PTW31014 chamber reduced the error from the calculated dose values.

The gamma pass rates largely depend on the type of verification tools. However, the same conclusion can be obtained using any of the three prescription dose verification tools for the $3 \% / 3 \mathrm{~mm}$ and $95 \%$ pass rate criteria, which are typically used for gamma index analysis. According to Reynolds et al. ${ }^{17)}$, as a result of verification of the delivered dose by treatment log-files from the RapidArc treatment using a linear accelerator, the pass rate was $98.3 \% \pm 1.4 \%$ for 
the acceptance criteria of $3 \% / 3 \mathrm{~mm}$, and it was evaluated to be the independent dose verification method.

Phantom-based patient-specific quality assurance is usually performed once before treatment; however, this cannot detect changes and dose delivery errors that occur throughout the entire process of the treatment. Therefore, it is suggested that real-time dose verification is required when treating patients. ${ }^{18)}$ However, Mobius3D is based on the treatment log-file, meaning it can be used as a simple and convenient tool to conduct quality assurance for each treatment without the time and space constraints of physical devices. As such, it is judged to be a suitable tool for prescription dose verification when the patient conditions are checked and adaptive radiotherapy is performed according to the change in conditions.

\section{Conclusions}

In this study, the feasibility of the Mobius3D dosimetry system, which has been used recently as a secondary tool for verifying prescribed dose, was examined. The calculation accuracy of the point dose using Mobius3D showed good agreement with the measured value within $2 \%$ on average. In addition, the Mobius3D result of gamma index analysis considering all criteria applied in this study showed consistent results with those obtained by EPID within $0.46 \% \pm 0.34 \%$ on average and within $3.14 \% \pm 2.85 \%$ for Octavius 4D. Therefore, we conclude that Mobius3D can be used interchangeably with phantom-based dosimetry systems, which are commonly used as patient-specific prescription dose verification tools, especially under the criteria of $3 \% / 3 \mathrm{~mm}$ and $95 \%$ pass rate.

\section{Acknowledgements}

This research was supported by Basic Science Research Program through the National Research Foundation of Korea (NRF) funded by the Ministry of Education (NRF2017R1D1A1B03027854).

\section{Conflicts of Interest}

The authors have nothing to disclose.

\section{Availability of Data and Materials}

All relevant data are within the paper and its Supporting Information files.

\section{References}

1. Wiggenraad RG, Petoukhova AL, Versluis L, van Santvoort JP. Stereotactic radiotherapy of intracranial tumors: a comparison of intensity-modulated radiotherapy and dynamic conformal arc. Int J Radiat Oncol Biol Phys. 2009;74: 1018-1026.

2. Wolden SL, Chen WC, Pfister DG, Kraus DH, Berry SL, Zelefsky MJ. Intensity-modulated radiation therapy (IMRT) for nasopharynx cancer: update of the Memorial Sloan-Kettering experience. Int J Radiat Oncol Biol Phys. 2006;64:57-62.

3. Park HJ, Griffin RJ, Hui S, Levitt SH, Song CW. Radiationinduced vascular damage in tumors: implications of vascular damage in ablative hypofractionated radiotherapy (SBRT and SRS). Radiat Res. 2012;177:311-327.

4. Létourneau D, Publicover J, Kozelka J, Moseley DJ, Jaffray DA. Novel dosimetric phantom for quality assurance of volumetric modulated arc therapy. Med Phys. 2009;36: 1813-1821.

5. Ezzell GA, Burmeister JW, Dogan N, LoSasso TJ, Mechalakos JG, Mihailidis D, et al. IMRT commissioning: multiple institution planning and dosimetry comparisons, a report from AAPM Task Group 119. Med Phys. 2009;36:5359-5373.

6. Low DA, Moran JM, Dempsey JF, Dong L, Oldham M. Dosimetry tools and techniques for IMRT. Med Phys. 2011;38: 1313-1338.

7. Dobler B, Streck N, Klein E, Loeschel R, Haertl P, Koelbl O. Hybrid plan verification for intensity-modulated radiation therapy (IMRT) using the 2D ionization chamber array I'mRT MatriXX--a feasibility study. Phys Med Biol. 2010;55: N39-N55.

8. Mans A, Remeijer P, Olaciregui-Ruiz I, Wendling M, Sonke JJ, Mijnheer B, et al. 3D Dosimetric verification of volumetric-modulated arc therapy by portal dosimetry. Radiother Oncol. 2010;94:181-187.

9. Ceberg S, Gagne I, Gustafsson H, Scherman JB, Korreman SS, Kjaer-Kristoffersen F, et al. RapidArc treatment verifi- 
cation in 3D using polymer gel dosimetry and Monte Carlo simulation. Phys Med Biol. 2010;55:4885-4898.

10. Hartmann B, Martisiková M, Jäkel O. Homogeneity of Gafchromic EBT2 film. Med Phys. 2010;37:1753-1756.

11. Childress N, Stevens E, Eklund D, Zhang M. Mobius3D white paper: dose calculation algorithm. Houston, TX: Mobius Medical Systems LP; 2012.

12. Qian J, Lee L, Liu W, Chu K, Mok E, Luxton G, et al. Dose reconstruction for volumetric modulated arc therapy (VMAT) using cone-beam CT and dynamic log files. Phys Med Biol. 2010;55:3597-3610.

13. Nelson CL, Mason BE, Robinson RC, Kisling KD, Kirsner SM. Commissioning results of an automated treatment planning verification system. J Appl Clin Med Phys. 2014; 15:4838.

14. Fontenot JD. Evaluation of a novel secondary check tool for intensity-modulated radiotherapy treatment planning. J Appl Clin Med Phys. 2014;15:4990.
15. Clemente-Gutiérrez F, Pérez-Vara C. Dosimetric validation and clinical implementation of two 3D dose verification systems for quality assurance in volumetric-modulated arc therapy techniques. J Appl Clin Med Phys. 2015;16: 5190.

16. McDonald DG, Jacqmin DJ, Mart CJ, Koch NC, Peng JL, Ashenafi MS, et al. Validation of a modern second-check dosimetry system using a novel verification phantom. J Appl Clin Med Phys. 2017;18:170-177.

17. Reynolds RR, Pompos A, Gu X, Jiang SB, Stojadinovic S. Initial experience with VMAT plan and Delivery verification using a DICOM-RT framework and Linac Delivery log files. Int J Radiat Oncol. 2014;90(1 Suppl):S886-S887.

18. Smith JC, Dieterich S, Orton CG. Point/counterpoint. It is still necessary to validate each individual IMRT treatment plan with dosimetric measurements before delivery. Med Phys. 2011;38:553-555. 